\title{
Structural and Functional Characterization of Covalently Modified Proteins Formed By
}

Glycating Agent, Glyoxal

Gurumayum Suraj Sharma ${ }^{1 \#}$, Reshmee Bhattacharya ${ }^{2 \#}$, Snigdha Krishna ${ }^{2}$, Suliman Y. Alomar $^{3}$, Afrah F. Alkhuriji ${ }^{4}$, Marina Warepam ${ }^{5}$, Kritika Kumari², Hamidur Rahaman ${ }^{5}$ \& Laishram Rajendrakumar Singh ${ }^{2 *}$

${ }^{1}$ Department of Botany, Bhaskaracharya College of Applied Sciences, University of Delhi, Delhi-110095, India

${ }^{2}$ Dr. B. R. Ambedkar Center for Biomedical Research, University of Delhi, Delhi-110007, India

${ }^{3}$ Doping Research Chair, Department of Zoology, College of Science, King Saud University, Riyadh- 11495, Saudi Arabia

${ }^{4}$ Department of Zoology, College of Science, King Saud University, Riyadh- 11495, Saudi Arabia

${ }^{5}$ Department of Biotechnology, Manipur University, Imphal, Manipur-795003, India.

\#These authors contributed equally to this manuscript.

*Corresponding Author: lairksingh@gmail.com (Dr. Laishram Rajendrakumar Singh) Mobile: +919811630757 

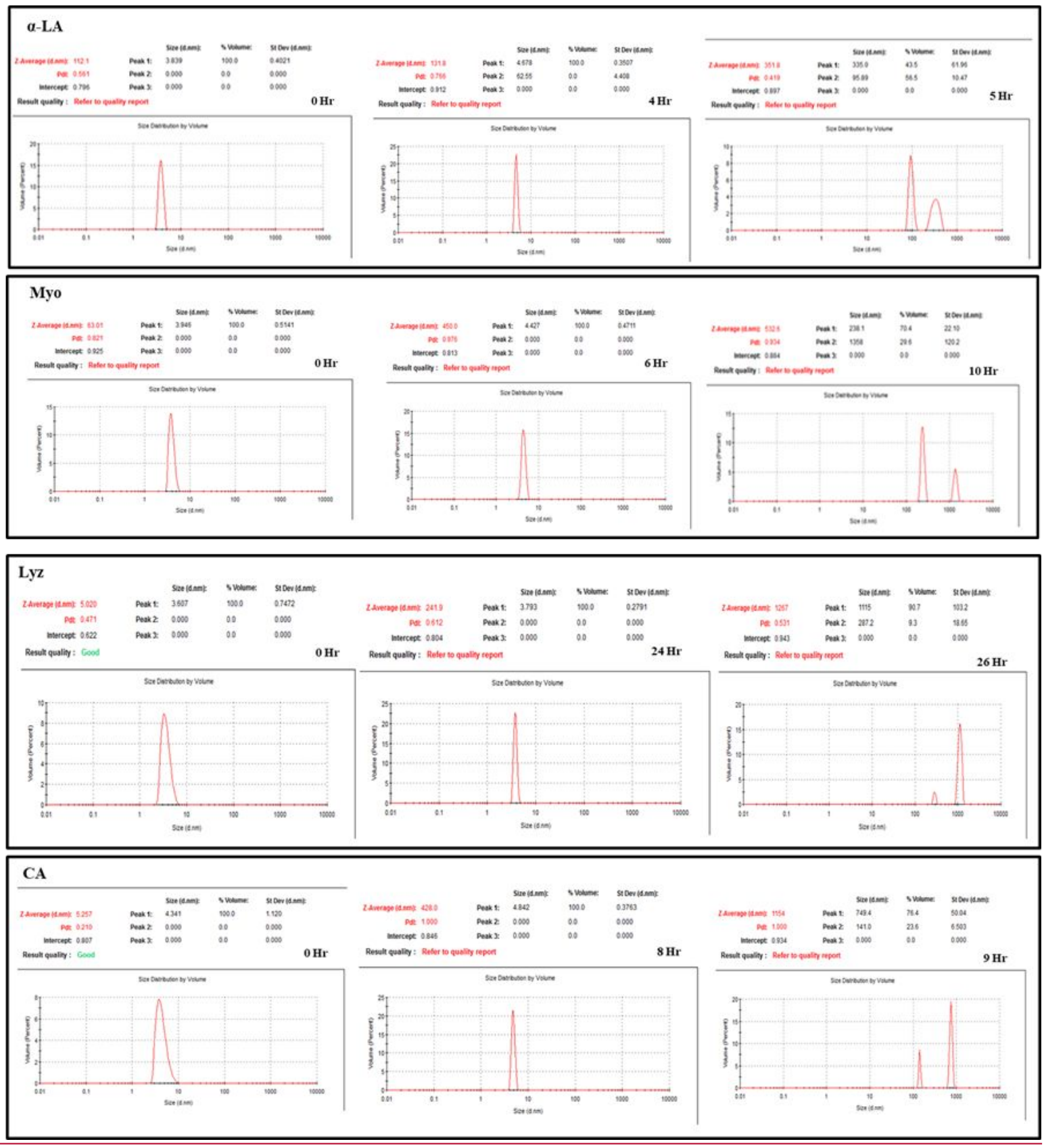


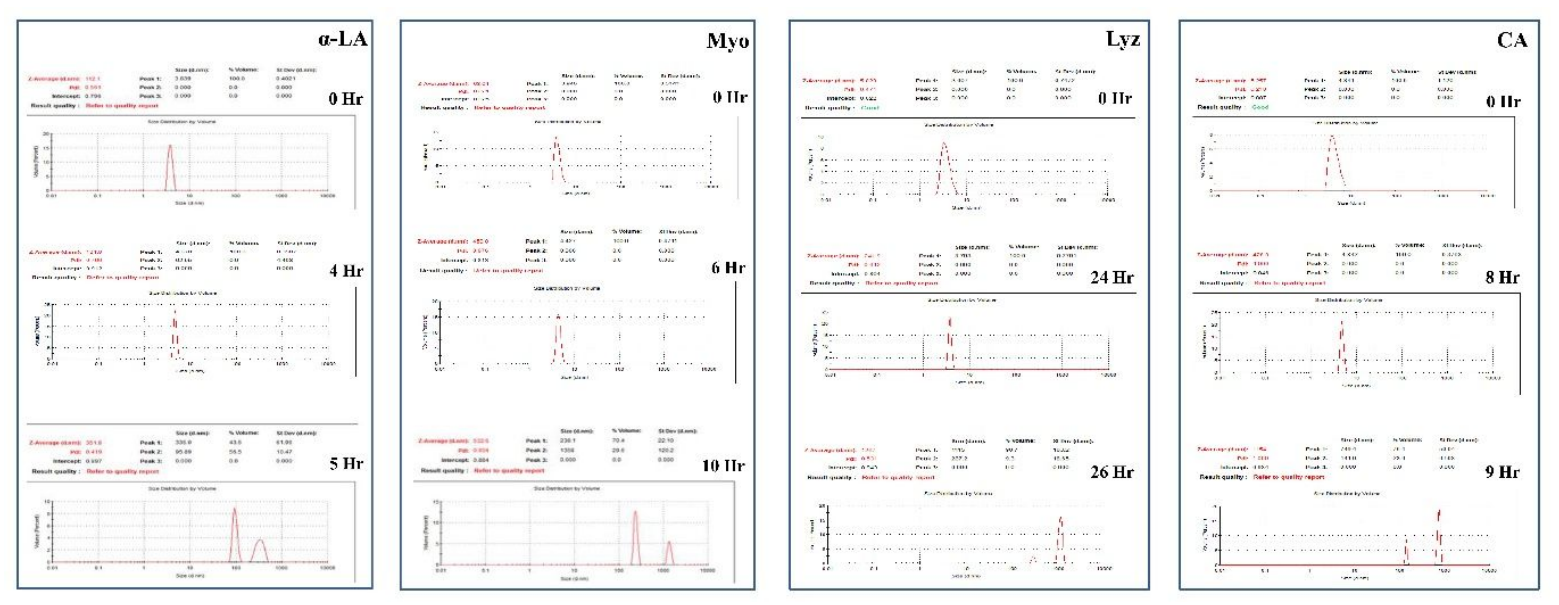

Figure S1: Size distribution by volume of $\alpha-\mathrm{LA}$, Myo, Lyz and CA upon treatment with $5 \mathrm{mM}$ Glyoxal obtained at different time frames.
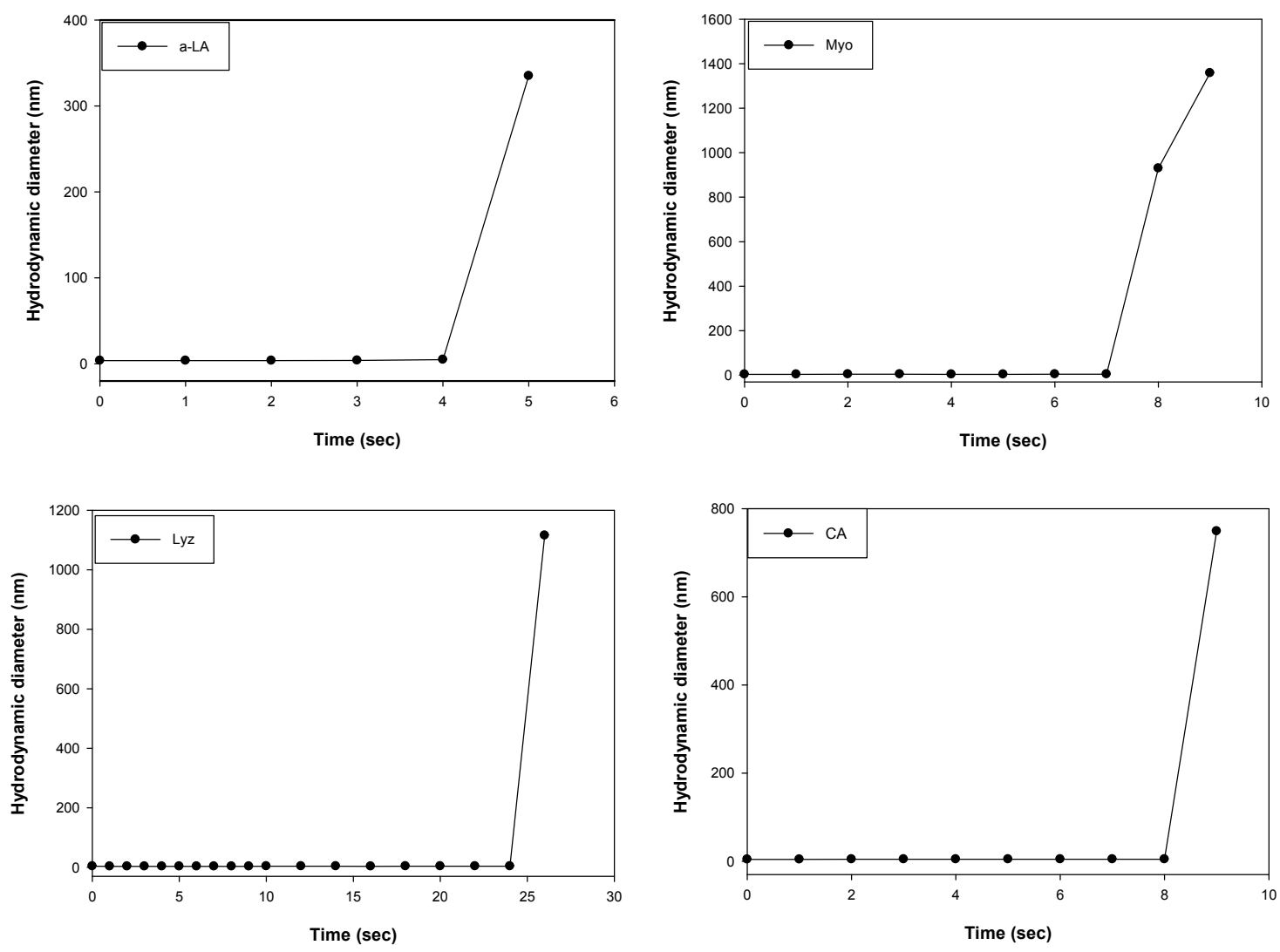

Figure S2: Time-dependent plot of hydrodynamic diameter $\alpha$-LA, Myo, Lyz and CA upon treatment with $5 \mathrm{mM}$ Glyoxal. 

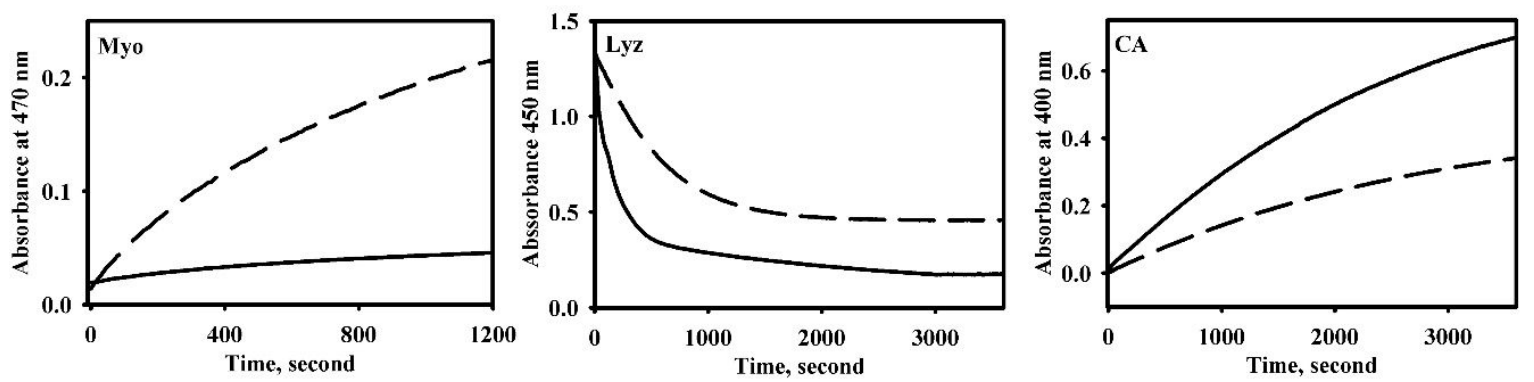

Figure S3: Activity assays of native and glycated enzymes. Time dependent kinetics for the hydrolysis of substrates by Myo, Lyz and CA.

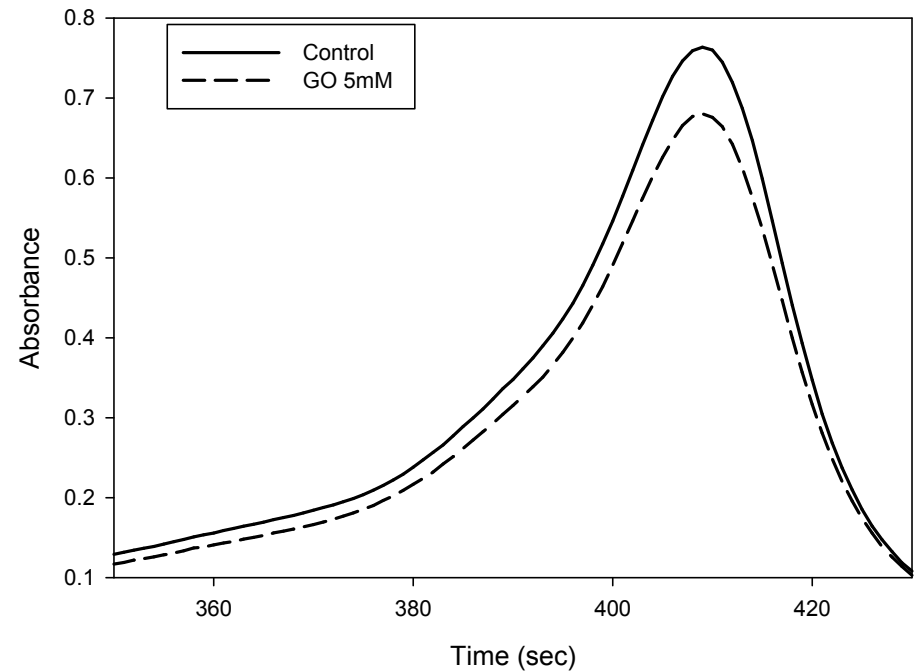

Figure S4: Heme absorbance measurement of Myo upon glycation. Visible absorption spectra of unmodified and modified Myo at $408 \mathrm{~nm}$.
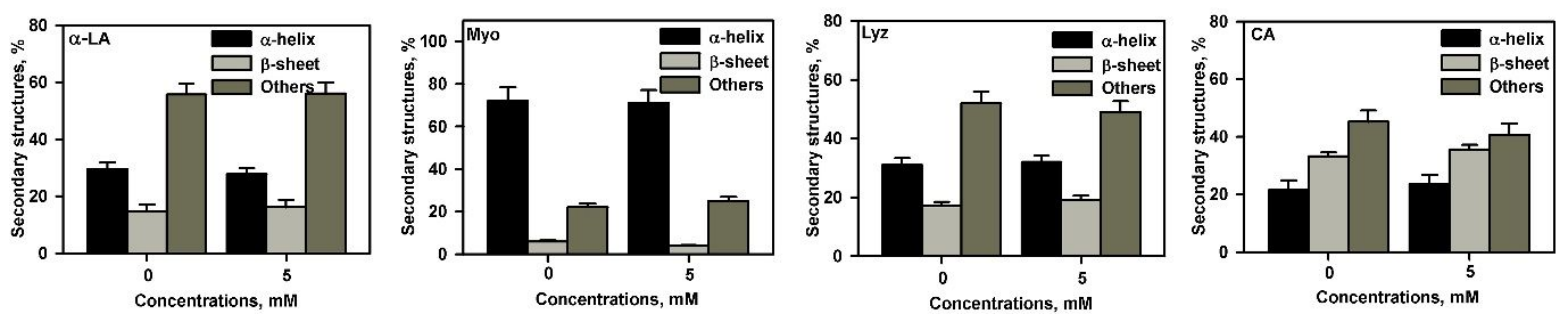

Figure S5: Secondary structural components of native and glycated proteins. 